\title{
Tuning p-Si(111) Photovoltage via Molecule|Semiconductor Electronic Coupling
}

\author{
Dylan G. Boucher ${ }^{\mathrm{a}}$, Kara Kearney ${ }^{\mathrm{b}}$, Elif Ertekin ${ }^{\mathrm{b}, \mathrm{c}}$ and Michael J. Rose ${ }^{\mathrm{a} *}$ \\ ${ }^{a}$ Department of Chemistry, The University of Texas at Austin \\ Austin, TX, 78712, USA \\ ${ }^{b}$ Department of Mechanical Science and Engineering, University of Illinois \\ Urbana, IL, 61801, USA \\ ${ }^{c}$ Institute for Carbon Neutral Energy Research (WPI-I2CNER) \\ Kyushu University, Japan
}

Contents

Page

1

XPS of Si(111)-AnthNO ${ }_{2}$ Surface

S-2

2

C 1s XPS

S-3

3

Photoconductivity Decay Traces

S-4

4

Illumination Intensity Dependence

S-5

5

Example DFT Structures

S-6

6

Total DOS

S-7

LDOS and Molecular DOS Comparison

S-8

8

Molecular HOMO-LUMO Visualization

S-10

Symmetry Adapted Correlation and MO Diagram

S-11 

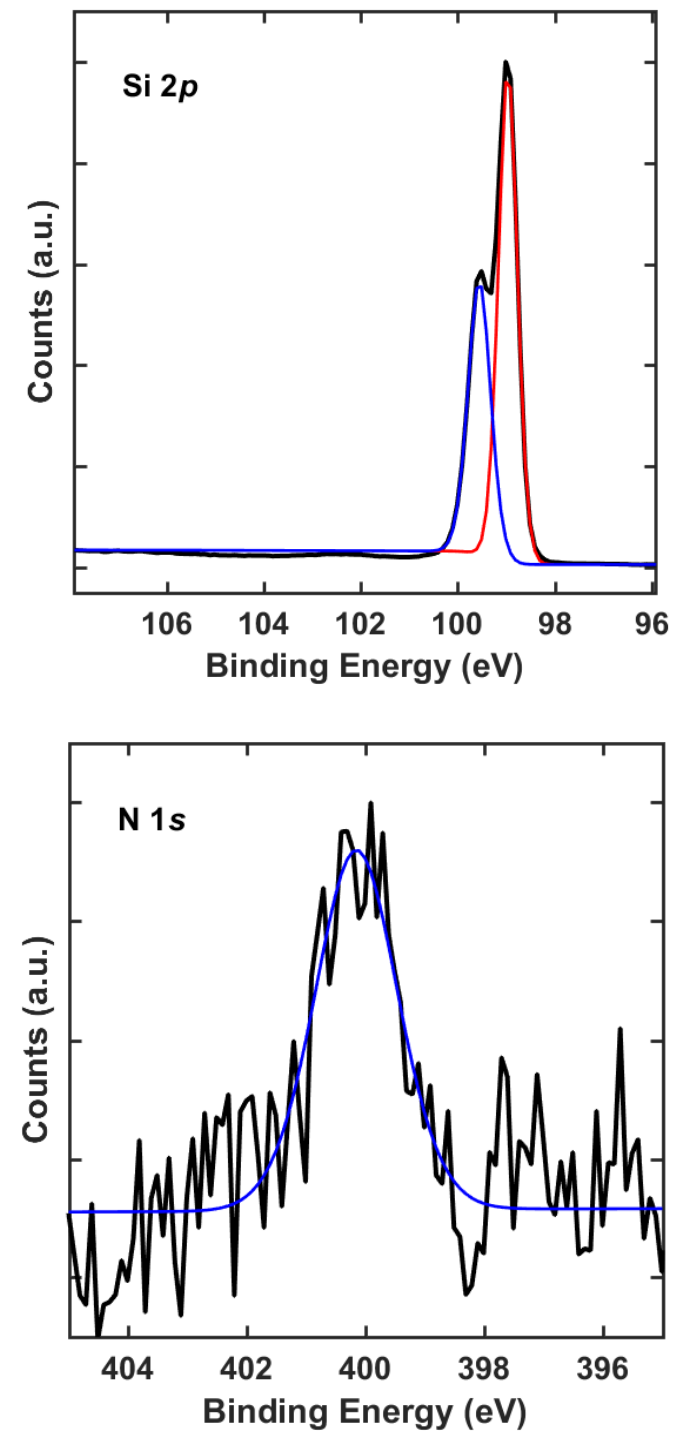

Figure S1. Representative XPS characterization of the $\mathrm{Si} 2 p$ region (top) and N $1 s$ region for the $\mathrm{Si}(111)-\mathrm{AnthNO}_{2}$ functionalized surface. 

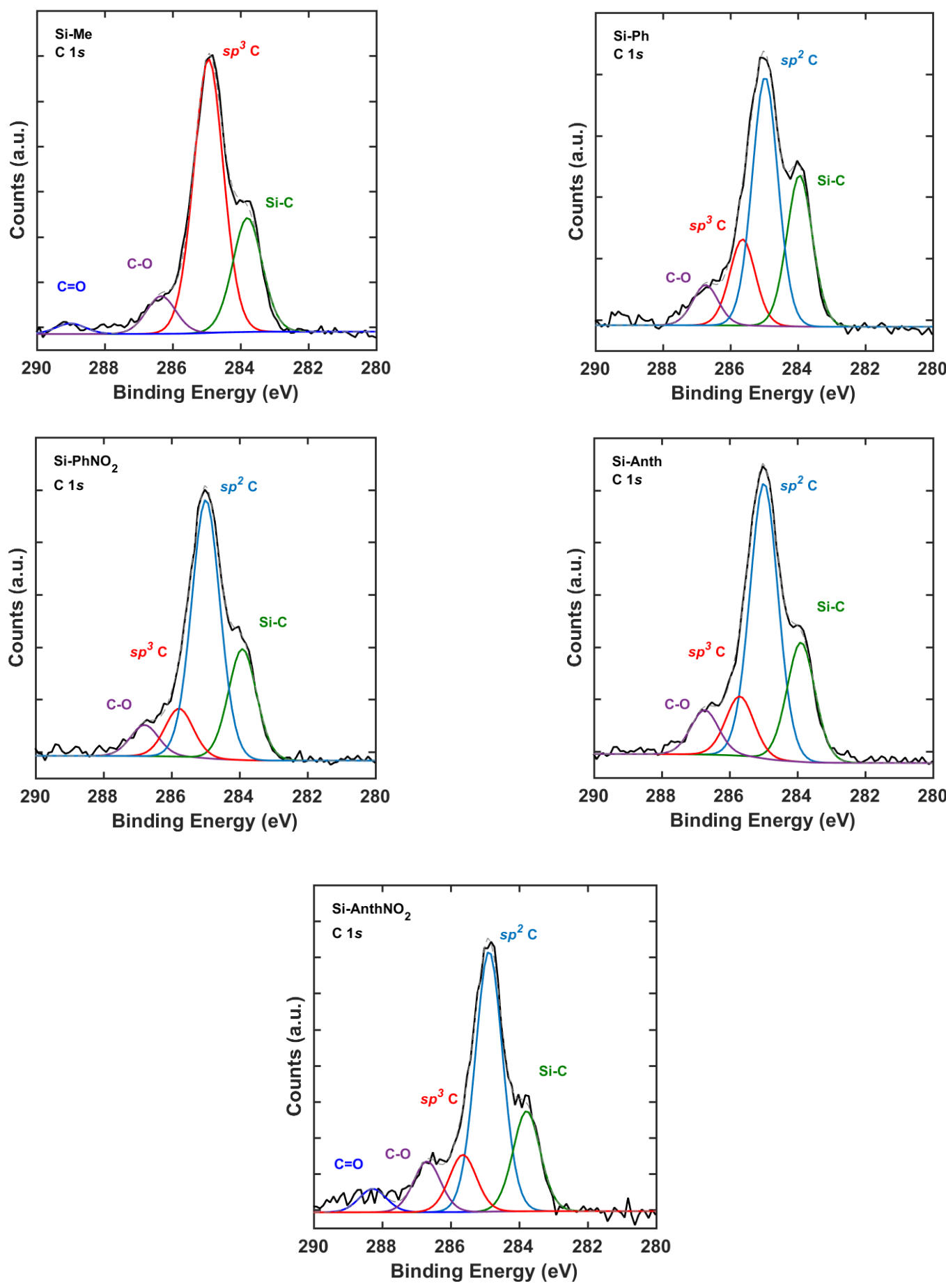

Figure S2. XP spectra of the $\mathrm{C} 1 s$ region for all surfaces. Carbon signals were fit with components constrained to known carbon chemical shifts: $\mathrm{C}=\mathrm{O}(288.9 \mathrm{eV}), \mathrm{C}-\mathrm{O}(286.7 \mathrm{eV}), \mathrm{sp}^{3} \mathrm{C}(285.7 \mathrm{eV}), \mathrm{sp}^{2} \mathrm{C}(284.9 \mathrm{eV})$, and Si-C $(283.9 \mathrm{eV})$. 


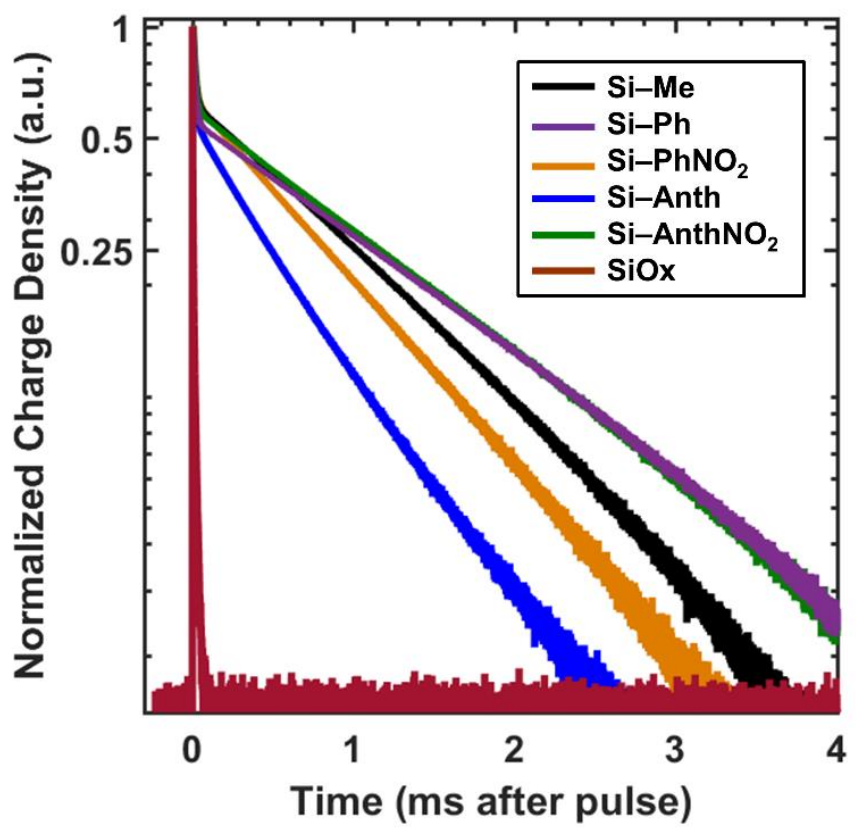

\begin{tabular}{lll}
\multicolumn{1}{c}{ Surface } & \multicolumn{1}{c}{$\begin{array}{c}\text { Lifetime } \\
\boldsymbol{\tau}(\mathbf{m s})\end{array}$} & $\boldsymbol{S}\left(\mathrm{cm} \mathrm{s}^{-\mathbf{1}}\right)$ \\
& $1.2 \pm 0.08$ & $22 \pm 2$ \\
Si-Me & $1.2 \pm 0.3$ & $22 \pm 6$ \\
$\mathbf{S i - P h}$ & $1.0 \pm 0.2$ & $27 \pm 6$ \\
Si-PhNO & \\
Si-Anth & $0.6 \pm 0.1$ & $39 \pm 7$ \\
Si-AnthNO $_{2}$ & $1.3 \pm 0.09$ & $19 \pm 0.9$ \\
SiO $_{x}$ & $0.006 \pm$ & $3945 \pm 52$ \\
& 0.0001 &
\end{tabular}

Figure S3. Representative photoconductivity decay traces after initial IR pulse for all surfaces and associated lifetimes $(\tau)$ and recombination velocities $(S)$. 


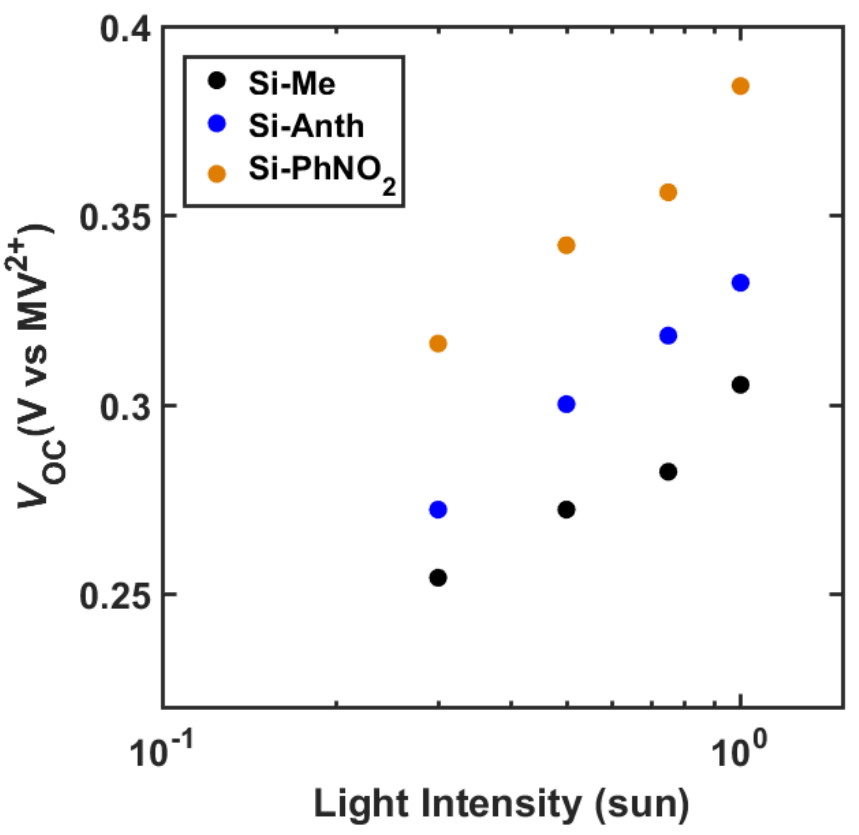

Figure S4. Light intensity dependence of select surfaces in contact with $\mathrm{MV}^{2+}$ on $V_{\mathrm{OC}}$. 
a)
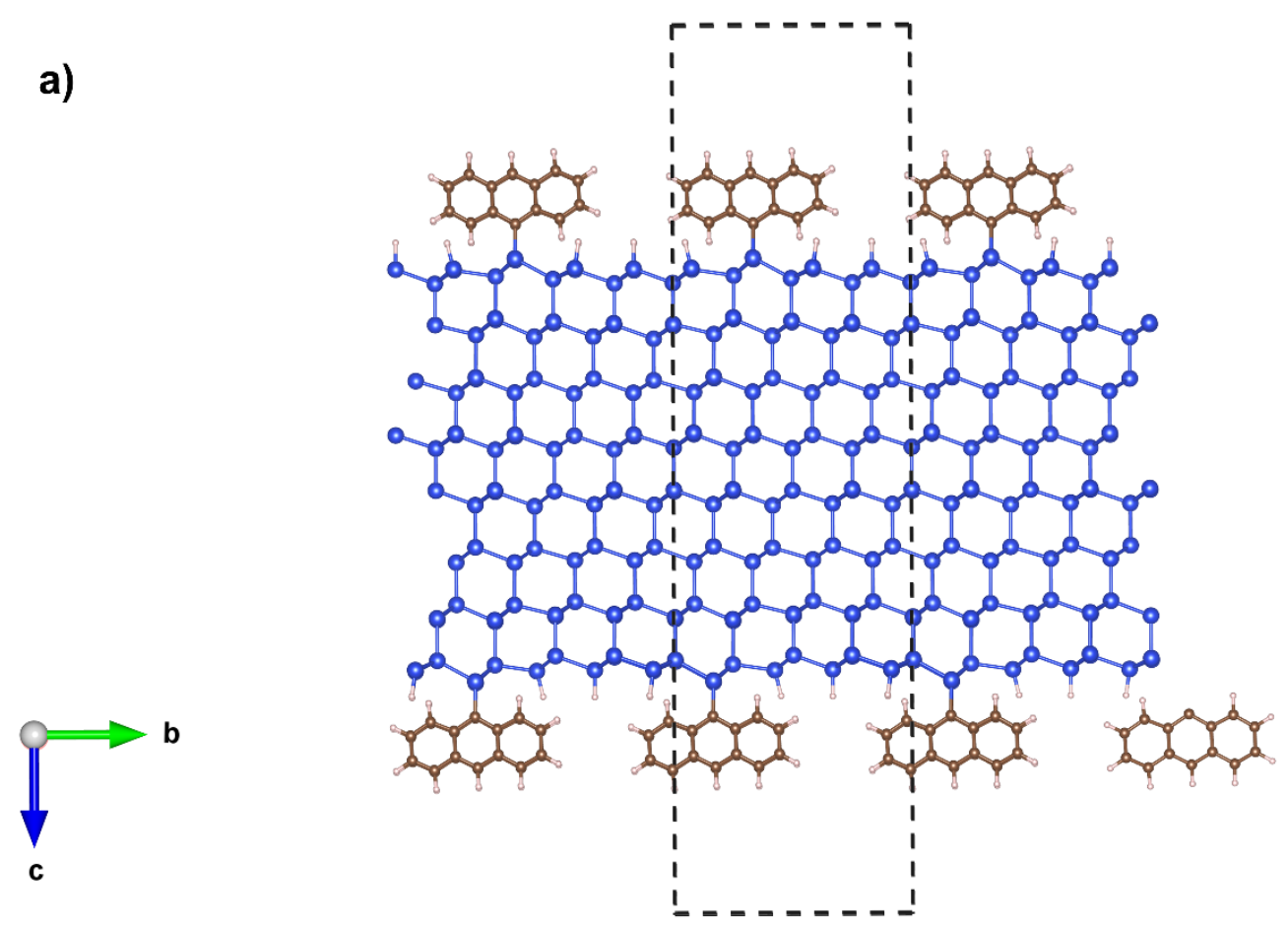

b)
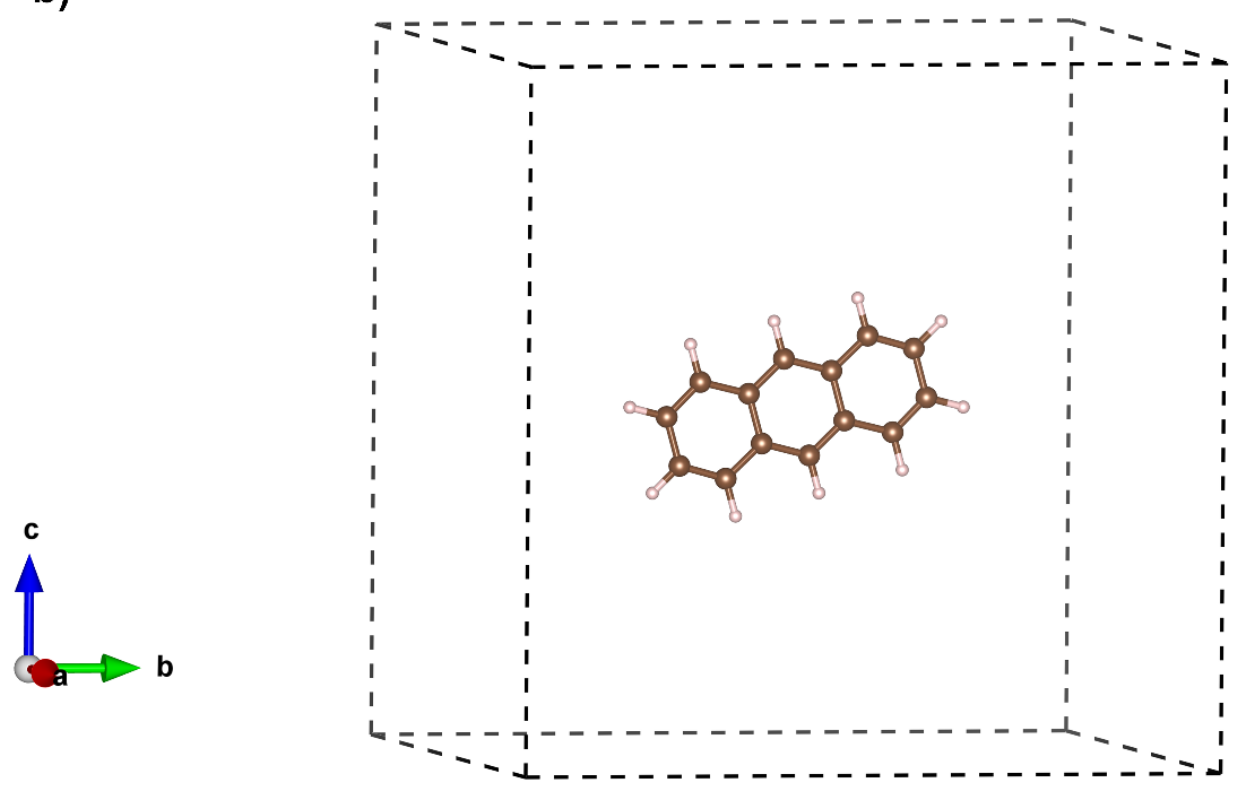

Figure S5. Example of $a$ ) converged Si(111) supercell with symmetric termination of functional groups and $b$ ) converged molecular structure. 
a)

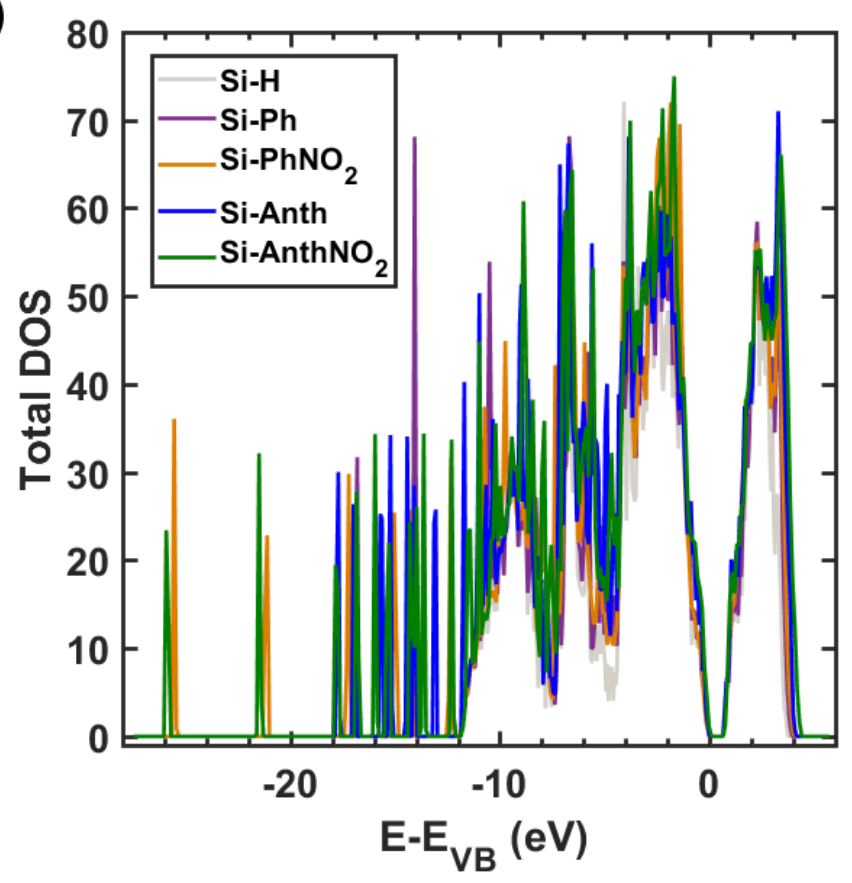

b)

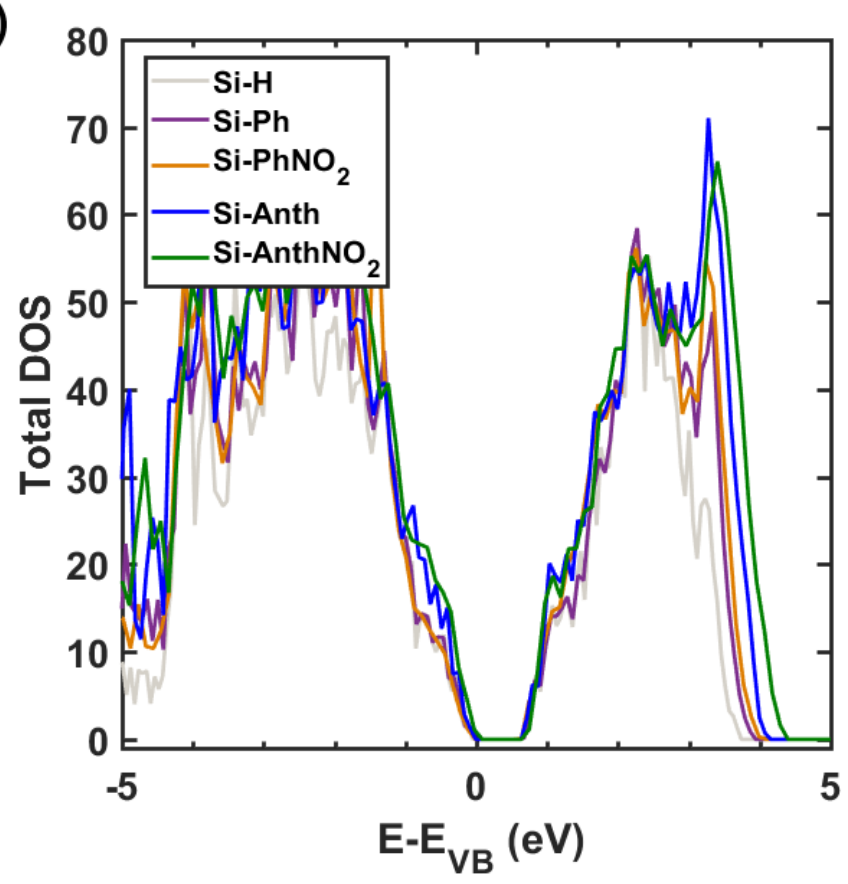

Figure S6. a) DFT calculated total density of states for functionalized surfaces and $b$ ) a zoomed in view of the total DOS around silicon band gap. 
a)

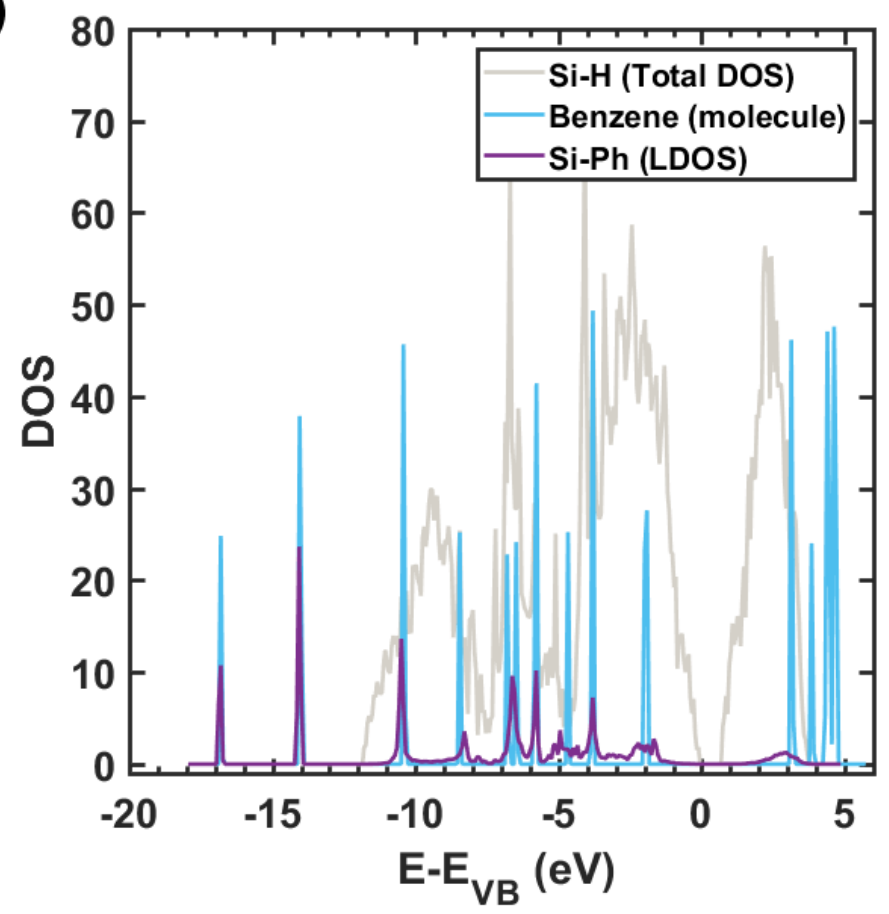

b)

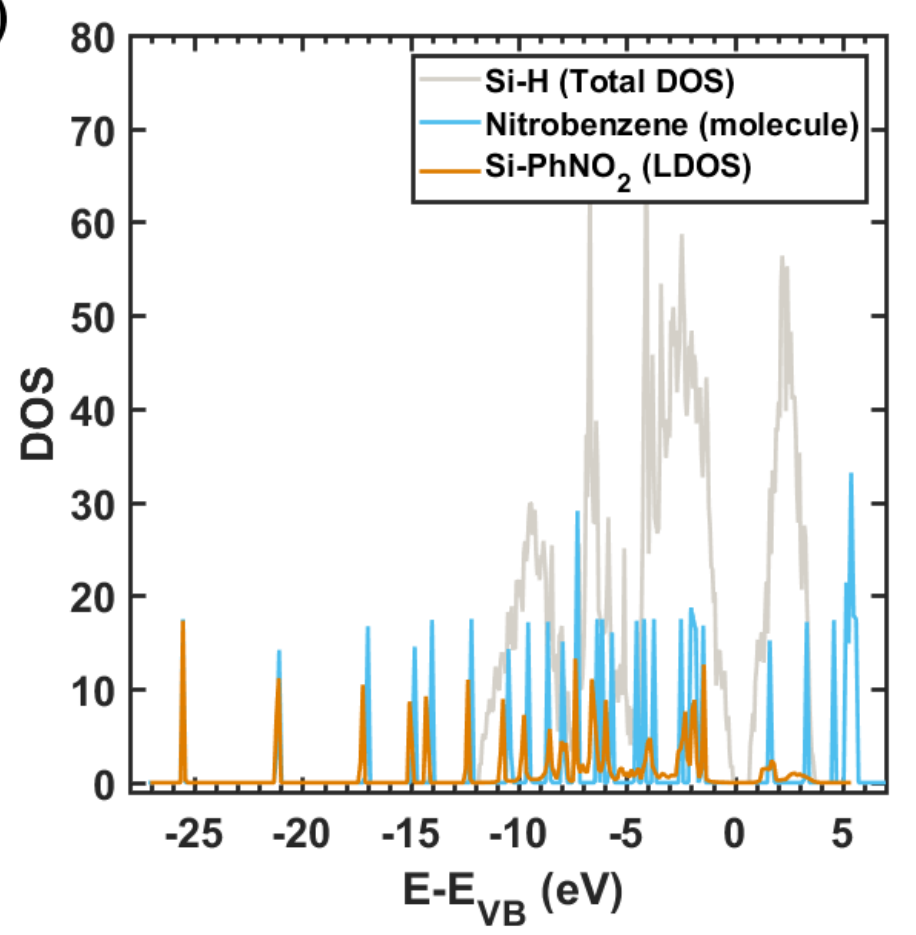



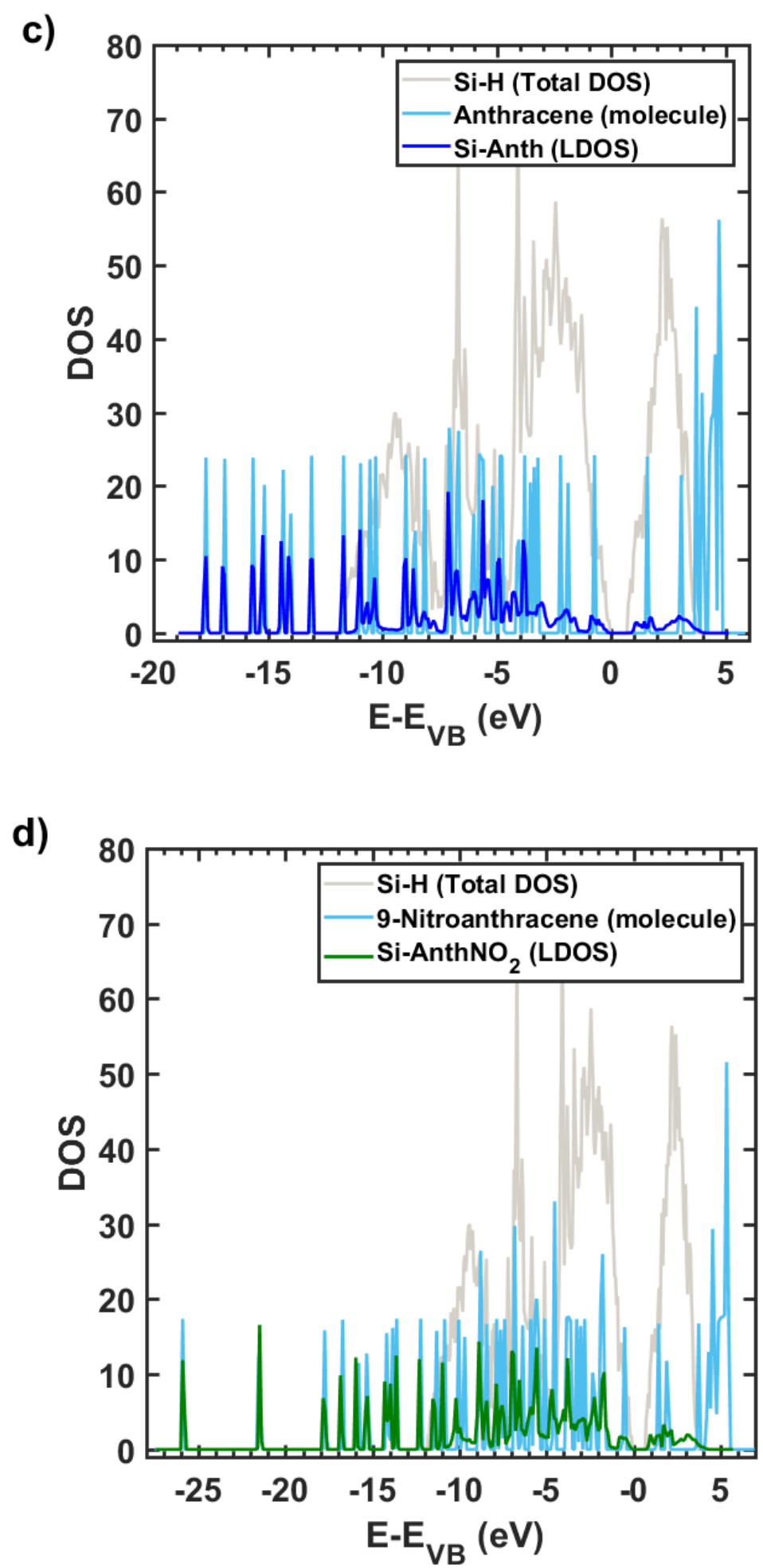

Figure S7. Comparison of the DFT calculated local density of states for functionalized surfaces with the density of states for the molecular analogue for $a$ ) $\mathrm{Si}-\mathbf{P h} b$ ) $\mathbf{S i}-\mathbf{P h N O}_{2}$ c) Si-Anth and $d$ ) Si-AnthNO2. The total DOS for Si$\mathbf{H}$ is given for reference in each case. 


\section{Molecule

a) Benzene

b) 4-Nitrobenzene
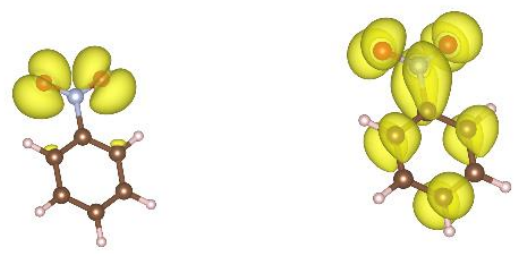

c) Anthracene
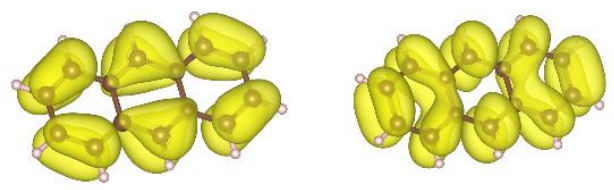

\section{d) 9-Nitroanthracene}
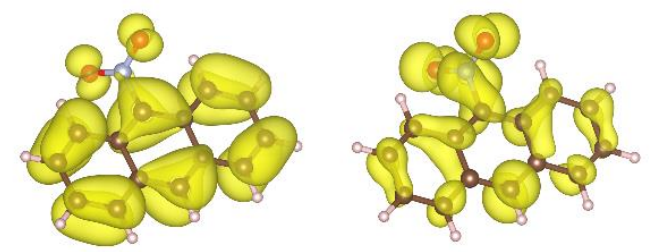

Figure S8. Visualization of the molecular orbitals rendered in VASP corresponding to the calculated DOS for the HOMO and LUMO states (combined electron density for spin-up and spin-down single-electron orbitals; hence, no phasing) for the isolated molecules. 


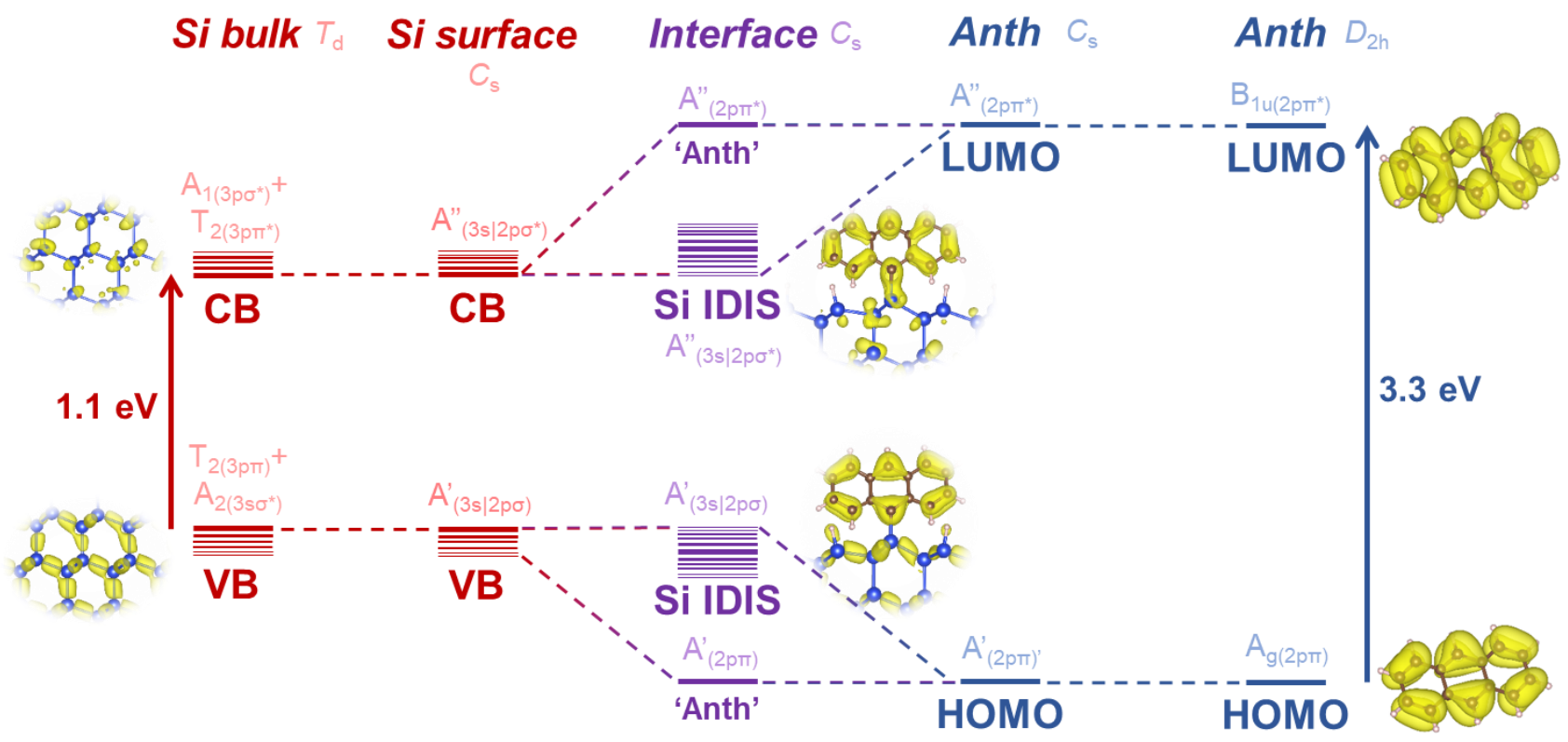

Figure S9. Full symmetry-adapted correlation diagram and MO representation for the orbital overlap and hybridization of exclusively molecular orbitals (Anth, far right side) and exclusively bulk silicon bands (Si bulk, far left side). Both Anth $\left(D_{2 \mathrm{~h}}\right)$ and bulk Si bulk $\left(T_{\mathrm{d}}\right)$ undergo well-established 'descent in symmetry' reductions to $C_{\mathrm{s}}$ (via $C_{2 \mathrm{v}}$ and $C_{3 \mathrm{v}}$, respectively - not shown) to ultimately enable symmetry-allowed orbital overlap in the localized $C_{\mathrm{s}}$ point group at the surface. 